\title{
Farnesylthiosalicylic Acid-Loaded Albumin Nanoparticle Alleviates Renal Fibrosis by Inhibiting Ras/RafI/p38 Signaling Pathway
}

\author{
Hui Huang, ',2 Qinhui Liu, ' \\ Ting Zhang, ${ }^{2}$ Jinhang Zhang, ${ }^{1,2}$ \\ Jian Zhou, ${ }^{1,2}$ Xiandan Jing, ${ }^{2}$ \\ Qin Tang,' Cuiyuan Huang, ',2 \\ Zijing Zhang,' Yingnan Zhao, ',2 \\ Guorong Zhang, 1,2 \\ Jiamin Yan, ${ }^{\text {,2 }}$ Yan Xia, ,,2 \\ Ying $\mathrm{Xu}$, ${ }^{\mathrm{,}, 2}$ jiahui Li, ${ }^{\mathrm{l}, 2}$ \\ Yanping $\mathrm{Li}^{\text {, ' Jinhan } \mathrm{He}^{\mathrm{l}, 2}}$ \\ 'Laboratory of Clinical Pharmacy and \\ Adverse Drug Reaction, State Key \\ Laboratory of Biotherapy, West China \\ Hospital, Sichuan University, Chengdu, \\ People's Republic of China; ${ }^{2}$ Department \\ of Pharmacy, State Key Laboratory of \\ Biotherapy, West China Hospital of \\ Sichuan University, Chengdu, People's \\ Republic of China
}

Background: Renal fibrosis is the common pathway in chronic kidney diseases progression to end-stage renal disease, but to date, no clinical drug for its treatment is approved. It has been demonstrated that the inhibitor of proto-oncogene Ras, farnesylthiosalicylic acid (FTS), shows therapeutic potential for renal fibrosis, but its application was hindered by the waterinsolubility and low bioavailability. Hence, in this study, we improved these properties of FTS by encapsulating it into bovine serum albumin nanoparticles (AN-FTS) and tested its therapeutic effect in renal fibrosis.

Methods: AN-FTS was developed using a classic emulsification-solvent ultrasonication. The pharmacokinetics of DiD-loaded albumin nanoparticle were investigated in SD rats. The biodistribution and therapeutic efficacy of AN-FTS was assessed in a mouse model of renal fibrosis induced by unilateral ureteral obstruction (UUO).

Results: AN-FTS showed a uniform spherical shape with the size of $100.6 \pm 1.12 \mathrm{~nm}$ and PDI < 0.25 . In vitro, AN-FTS displayed stronger inhibitory effects on the activation of renal fibroblasts cells NRK-49F than free FTS. In vivo, AN-FTS showed significantly higher peak concentration and area under the concentration-time curve. After intravenous administration to UUO-induced renal fibrosis mice, AN-FTS accumulated preferentially in the fibrotic kidney, and alleviated renal fibrosis and inflammation significantly more than the free drug. Mechanistically, the improved anti-fibrosis effect of AN-FTS was associated with greater inhibition in renal epithelial-to-mesenchymal transformation process via Ras/Rafl/p38 signaling pathway.

Conclusion: The study reveals that AN-FTS is capable of delivering FTS to fibrotic kidney and showed superior therapeutic efficacy for renal fibrosis.

Keywords: albumin nanoparticle, epithelial-mesenchymal transition, farnesylthiosalicylic acid, Ras, renal fibrosis

\section{Introduction}

Laboratory of Clinical Pharmacy and

Adverse Drug Reaction, State Key

Laboratory of Biotherapy, West China

Hospital of Sichuan University, Chengdu,

Sichuan, People's Republic of China

Tel +86-28-85 I 64I 28

Email liyanping_512@163.com

Jinhan $\mathrm{He}$

Department of Pharmacy, State Key

Laboratory of Biotherapy, West China

Hospital of Sichuan University, Chengdu,

Sichuan, People's Republic of China

Tel +86-28-85426416

Email jinhanhe@scu.edu.cn
Chronic kidney disease (CKD) is a serious problem and challenge to human health with a $10 \%$ global prevalence. ${ }^{1}$ Almost all forms of CKDs often lead to renal fibrosis, which is characterized by numerous inflammatory cells infiltration, myofibroblast activation and excessive extracellular matrix accumulation. ${ }^{2}$ Without treatments, renal fibrosis may further develop into end-stage renal diseases and eventually require dialysis or kidney transplantation. ${ }^{3}$ To date, few drugs have been approved for clinic treatment of renal fibrosis. Thus, it is urgently needed to develop effective anti-renal fibrotic drugs and appropriate drug delivery systems for renal fibrosis treatment. 
The pathogenesis of renal fibrosis is very complicated, among which epithelial mesenchymal transition (EMT) plays an important role in its occurrence and progression. ${ }^{4}$ During the EMT process, mesenchymal markers such as vimentin and snail gradually substitute epithelial markers such as E-cadherin and occludins. ${ }^{5}$ As an essential profibrotic cytokine, transforming growth factor- $\beta$ (TGF- $\beta$ ) is a key regulator in the renal EMT process. ${ }^{6}$ Interestingly, the proto-oncogene Ras is crucial for the TGF- $\beta 1$-induced EMT in renal fibrosis. ${ }^{7}$ Ras has three major isoforms: H-Ras, $\mathrm{K}-\mathrm{Ras}$, and N-Ras, and they share a high degree of sequence homology. ${ }^{8}$ Accumulating evidence has demonstrated that Ras family plays an important role in renal fibrosis. ${ }^{9}$ In animal models, RAS was significantly up-regulated in renal tubulointerstitial fibrosis caused by unilateral ureteral obstruction (UUO). ${ }^{9,10}$ Knockdown of K-Ras by antisense oligonucleotide prevented renal fibrosis in folate-induced CKD mice. ${ }^{11}$ In H-Ras knockout mice, UUO induced lower EMT and renal interstitial fibrosis. ${ }^{10}$ EMT in the progression of renal fibrosis can be suppressed by inhibiting the transmission of Ras/Raf1/p38 MAPK signaling pathway. ${ }^{7,12}$ Thus, pharmacological inhibition of Ras may have promise for the treatment of renal fibrosis.

S-trans, trans-farnesylthiosalicylic acid (FTS) is a specific inhibitor of proto-oncogene Ras. ${ }^{13}$ The structure of FTS is similar to the carboxy-terminal structure of Ras protein, and it can be used as a part of the recognition unit to inhibit the activity of Ras protein. ${ }^{14}$ FTS has been reported to inhibit the growth of H-Ras and K-Ras transformed fibroblasts in vitro. ${ }^{10,11}$ FTS has also been shown to alleviate inflammation and fibrosis in chemically induced liver fibrosis in rats. ${ }^{15,16}$ FTS could suppress TGF- $\beta 1$ induced renal fibroblasts NRK-49F activation by inhibiting the EMT process. Furthermore, FTS reduced glomerular cellular proliferation and macrophage infiltration in rat Thy- 1 nephritis. ${ }^{17}$ These studies suggest that FTS may have therapeutic potential in treating renal fibrosis. However, several unfavorable features of FTS, such as water-insolubility and low bioavailability, have greatly hindered its clinical application.

Numerous studies show that nanoparticles have been widely used in drug delivery because they can solve the problems of therapeutic agents including poor solubility, low bioavailability and lack of target ability. ${ }^{18}$ Albumin, a multifunctional protein carrier, has been shown to be nontoxic, non-immunogenic, biocompatible and biodegradable. ${ }^{19}$ Thus, it is an ideal material to prepare nanoparticles for drug delivery. Since the albumin-bound paclitaxel nanoparticle was approved by US Food and
Drug Administration in $2005,{ }^{20}$ albumin nanoparticles have drawn considerable attention due to their high binding capacity with various drugs and well tolerated without any serious side-effects. ${ }^{21}$ Recently, Guo et al have shown that albumin nanoparticles with a particle size of $70-130 \mathrm{~nm}$ can passively target the kidney. ${ }^{22}$ Therefore, to tackle the poor water solubility and low bioavailability of FTS, we select albumin nanoparticles (AN) with defined sizes to deliver FTS selectively to the kidney.

Using a classic emulsification-solvent ultrasonication method, we prepared FTS-loaded albumin nanoparticles (ANFTS) with the size of about $100 \mathrm{~nm} .{ }^{22}$ Our results showed that encapsulating FTS into albumin nanoparticles greatly improved its pharmacokinetic properties and selectively delivery to fibrotic kidney. After administration to UUO-induced renal fibrosis mice intravenously, AN-FTS alleviated renal injury and fibrosis significantly than free drug via inhibition of Ras/Rafl/p38 MAPK signaling pathway.

\section{Materials and Methods Materials}

S-trans, trans-farnesylthiosalicylic acid (FTS) and soybean oil was obtained from Aladdin (Shanghai, China). Bovine serum albumin (BSA) was purchased from Biofrox GmbH (Hamburg, Germany). 1,1'-dioctadecyl-3,3,3',3'tetramethylindodicarbo-cyanine (DiD) was provided by Biotium (Hayward, USA). All other chemical reagents and solvents were purchased from Sigma-Aldrich (St. Louis, USA) and are analytically pure or greater.

\section{Animals}

Male C57BL/6J mice (8-10 weeks of age, 18-25 g) and male SD rats $(180-220 \mathrm{~g})$ were purchased from Beijing HFK bioscience Co., Ltd (China) and kept in a specific pathogen-free animal house in West China Hospital of Sichuan University. All animal procedures were performed under ethical approval by the Institutional Animal Care and Use Committee of Sichuan University, Chengdu, China, and conform to the Guide for the Care and Use of Laboratory Animals published by the United States National Institutes of Health.

\section{Preparation of FTS-Loaded Albumin Nanoparticles}

FTS-loaded albumin nanoparticles (AN-FTS) were prepared using an emulsification-solvent ultrasonication method as previously described. ${ }^{22}$ Briefly, $4 \mathrm{mg}$ of FTS and $25 \mathrm{mg}$ of 
soybean oil were dissolved in a $0.5 \mathrm{~mL}$ ethyl acetate and dichloromethane $(2: 1, \mathrm{v} / \mathrm{v})$ mixture solvent to obtain the organic phase. Then, $5 \mathrm{~mL}$ of $2 \%(\mathrm{w} / \mathrm{v})$ BSA in distilled water was added to the organic phase. The mixture was intermittently ultrasonicated at $330 \mathrm{~W}$ for $8 \mathrm{~min}$ in an ice water bath. The organic solvent was removed by vacuum rotary evaporation under $35^{\circ} \mathrm{C}$, and then the nanoparticles were concentrated using an ultrafiltration tube with a retained molecular weight of $30 \mathrm{kDa}$. The concentrated nanoparticles were diluted by $5 \%$ glucose solution to $1 \mathrm{~mL}$ to obtain the final AN-FTS. DiD-loaded albumin nanoparticles (AN-DiD) were prepared using the same method with the exception that FTS was substituted by DiD.

\section{Characterization of AN-FTS}

The particle size and zeta potential of AN-FTS were determined by dynamic light scattering and electrophoretic light-scattering technology, respectively (Zetasizer Nano ZS90 instrument, Malvern, UK). After being stained with $2 \%$ phosphotungstic acid, AN-FTS samples were placed on copper grids with films for transmission electron microscopy (H-600, Hitachi, Japan).

The encapsulation efficiency (EE\%) and drug loading capacity (DLC\%) of AN-FTS were determined using an ultrafiltration method. ${ }^{22}$ Briefly, free FTS was removed by ultrafiltration, then the concentration of encapsulated FTS was measured by high-performance liquid chromatography (HPLC) on an Agilent 1260 system (Agilent, USA) with a C18 column $(5 \mu \mathrm{m}, 15 \mathrm{X} 4.6 \mathrm{~mm})$ at $262 \mathrm{~nm} .{ }^{23}$ The mobile phase was methanol/water $(90 / 10, \mathrm{v} / \mathrm{v})$ containing $0.1 \%$ glacial acetic acid, which was used at a flow rate of $1.0 \mathrm{~mL} / \mathrm{min}$. Then $\mathrm{EE} \%$ and DLC\% were calculated using the following equations: $\mathrm{EE} \%=$ (weight of the drug in nanoparticles/ weight of the drug added $) \times 100 \%$, DLC $\%=$ (weight of the drug in nanoparticles /total weight of nanoparticles) $\times 100 \%$.

\section{In vitro Release of FTS from AN-FTS}

The in vitro release profile of FTS from AN-FTS was determined using the dynamic dialysis method. ${ }^{24}$ Briefly, $1 \mathrm{~mL}$ of free FTS or AN-FTS (FTS concentration $=1 \mathrm{mg}$ / $\mathrm{mL}$ of) was added into a dialysis bag with a retained molecular weight of $8000-14,000 \mathrm{Da}$, which was then immersed into $4 \mathrm{~mL}$ of PBS (pH 7.4) containing $0.2 \%$ Tween 80 and shaken in a horizontal shaker $\left(37{ }^{\circ} \mathrm{C}\right.$, $70 \mathrm{rpm})$. After that, at predetermined time points, the dialysates were withdrawn and replaced with $4 \mathrm{~mL}$ of fresh medium at fixed time points. The samples were diluted with methanol followed by FTS quantification with HPLC method as described above.

\section{Cell Culture and Treatments}

Normal rat renal fibroblasts NRK-49F cells, obtained from American Type Culture Collection (ATCC, Maryland, USA), were cultured in Dulbecco's modified Eagle's medium (Gibco, USA) containing 10\% fetal bovine serum (Hyclone, USA), $100 \mu \mathrm{g} / \mathrm{mL}$ streptomycin and $100 \mathrm{U} / \mathrm{mL}$ penicillin. To test the effect of AN-FTS on TGF- $\beta 1$-induced activation of NRK-49F, the cells were exposed to free FTS or AN-FTS (20 uM of FTS) containing $2 \mathrm{ng} / \mathrm{mL}$ of TGF- $\beta 1$ (Peprotech, USA) for $48 \mathrm{~h}$, then harvested for analysis.

\section{Pharmacokinetic Study of AN-DiD in Rats}

Pharmacokinetic study of AN-FTS was performed in SD rats as previously described. ${ }^{25}$ Briefly, SD rats were randomly divided into two groups and injected intravenously with free DiD or AN-DiD (200 $\mu \mathrm{g} / \mathrm{kg}$ DiD). At predetermined time points, blood was collected from fundus veins and diluted with acetone, and then determined by a multifunctional microplate reader (Thermo Fisher Scientific, USA). The pharmacokinetics parameters were analyzed with software DAS 2.0.

\section{Unilateral Ureteral Obstruction}

Unilateral ureteral obstruction (UUO) was performed in C57BL/6J mice to simulate the pathologic status of clinical patients with ureteral obstruction. ${ }^{26,27}$ Briefly, animals were anesthetized with inhaled isoflurane, and the right kidney was exposed by surgical scissors. The right ureter was carefully separated and blocked by two-point ligations with 5-0 silk sutures. The abdominal incision was closed with $0-4$ silk sutures. Sham-operated mice were performed all the same operations, except their right ureter was not ligated.

\section{Biodistribution of AN-DiD in UUO-Induced Mice}

UUO mice were randomly divided into two groups, one group received free DiD dissolved in 5\% DMSO and another group received AN-DiD via the tail vein $(200 \mu \mathrm{g}$ $\mathrm{DiD} / \mathrm{kg}$ ). After $4 \mathrm{~h}$, the mice were euthanized, the major organs were collected and visualized with an in vivo imaging system (Quick View 3000, Bio-Real, Austria). The mean fluorescence intensity in kidney was determined based on the semi-quantitative analysis of the ex vivo fluorescent images. 


\section{Therapeutic Efficacy of AN-FTS in UUO-Induced Renal Fibrosis Mice}

C57BL/6J mice were conducted UUO surgery as described above. On the second day of surgery, mice were treated with blank solvent (vehicle), blank albumin nanoparticles (blank AN), free FTS or AN-FTS every day via the tail vein at a dosage of $20 \mathrm{mg}$ per $\mathrm{kg}$. On day 8 after UUO, mice were sacrificed, and serum and tissues were collected for further determination.

Serum creatinine, urea nitrogen, and renal hydroxyproline content were assayed using commercial kits ((Nan Jing Jian Cheng Biochemical Institute, Nanjing, China) according to the manufacturer's instructions. For histology, kidney tissues were sectioned into $4 \mu \mathrm{m}$ thick, followed by hematoxylin and eosin (H\&E), periodic acid-Schiff (PAS), and Masson's trichrome staining. The results were observed with an optical microscope (Nikon, Tokyo, Japan) and the images were captured.

\section{Immunohistochemistry}

Kidney tissues were fixed in 10\% formalin, embedded in paraffin, sectioned into $4 \mu \mathrm{m}$ per piece. Antigen recovery were performed in EDTA buffer $\left(\mathrm{pH} \mathrm{8.0)}\right.$ at $98{ }^{\circ} \mathrm{C}$ for 30 min. After natural cooling, endogenous peroxidase was blocked with $3 \% \mathrm{H}_{2} \mathrm{O}_{2}$ in methanol. The sections were incubated with rabbit antibodies against $\alpha$-SMA (HuaBio, China) or Ras (Boster, China) overnight at $4{ }^{\circ} \mathrm{C}$, then with horseradish peroxidase-conjugated secondary antibody (Jackson ImmunoResearch, USA) for $1 \mathrm{~h}$ at room temperature. Nuclei were stained with hematoxylin.

\section{Real-Time PCR Analysis}

Total RNA was extracted from kidney tissues or NRK-49F cells using Trizol (ApplyGen, Beijing, China) and reversetranscribed into complementary DNA using a commercial kit (TaKaRa, Japan). Real-time RT-PCR was performed as previously described. $^{25}$ The primers are listed in Supplementary Table 1.

\section{Western Blot Analysis}

Total protein was extracted from kidney tissues or NRK49F cells. Western blot analysis was conducted as described in previous study. ${ }^{25}$ The antibodies are listed in Supplementary Table 2.

\section{Statistical Analysis}

All data are presented as mean \pm SEM. Statistical significance was determined by Student's unpaired two-tailed $t$-test or one-way ANOVA for multiple comparison tests. $P$ values $<0.05$ and $<0.01$ were considered indications of significant difference and extremely significant difference, respectively.

\section{Result}

\section{Preparation and Characterization of AN-FTS}

AN-FTS was prepared by an emulsification-solvent ultrasonication method as previously described. ${ }^{22}$ Dynamic light scattering (DLS) indicated the average size of blank $\mathrm{AN}$ and AN-FTS was about $100 \mathrm{~nm}$ with polydispersity index (PDI) below 0.25, confirming size homogeneity (Figure 1A). Transmission electron microscopy exhibited albumin nanoparticles had uniform spherical morphology, and the size of AN-FTS was consistent with DLS data (Figure 1A). Based on the HPLC assay, AN-FTS had an $\mathrm{EE} \%$ of $98.6 \pm 1.2 \%$ and DLC\% of $2.31 \pm 0.15 \%$ for the drug (Table 1). Additionally, no significant size or PDI changes were found when leaving the albumin nanoparticles at $4{ }^{\circ} \mathrm{C}$ for 14 days, indicating the intrinsic stability of AN-FTS (Figure 1B).

The release profiles of free FTS and AN-FTS was investigated in PBS ( $\mathrm{pH}$ 7.4) using the dialysis method. In contrast to the rapid release of free FTS, AN-FTS showed sustained release of FTS. In particular, approximately $35 \%$ of the entrapped drug in AN-FTS was released from dialysis bags, compared to over $80 \%$ of the free FTS within $12 \mathrm{~h}$ (Figure 1C).

\section{AN-FTS Inhibits NRK-49F Activation via Suppressing Ras Expression}

The activation of myofibroblast is the central link for renal fibrosis. $^{28}$ To test the anti-fibrosis efficacy of AN-FTS in vitro, normal rat renal fibroblast NRK-49F cells were stimulated with TGF- $\beta 1$, the most important inducer for myofibroblast activation. ${ }^{29}$ As expected, TGF- $\beta$-treated NRK-49F cells showed significantly enhanced mRNA expression of fibrotic genes including $\alpha$-SMA, Collagen, Pai-1, and fibronectin (Figure 2A). This upregulation was slightly decreased by free FTS. In contrast, AN-FTS substantially inhibited the expression of fibrotic genes induced by TGF- $\beta 1$ at the same concentration of free FTS. Consistently, treating NRK-49F cells with AN-FTS reduced the protein levels of $\alpha$-SMA, Colla1, and fibronectin significantly than free FTS (Figure 2B). Neither vehicle nor blank 

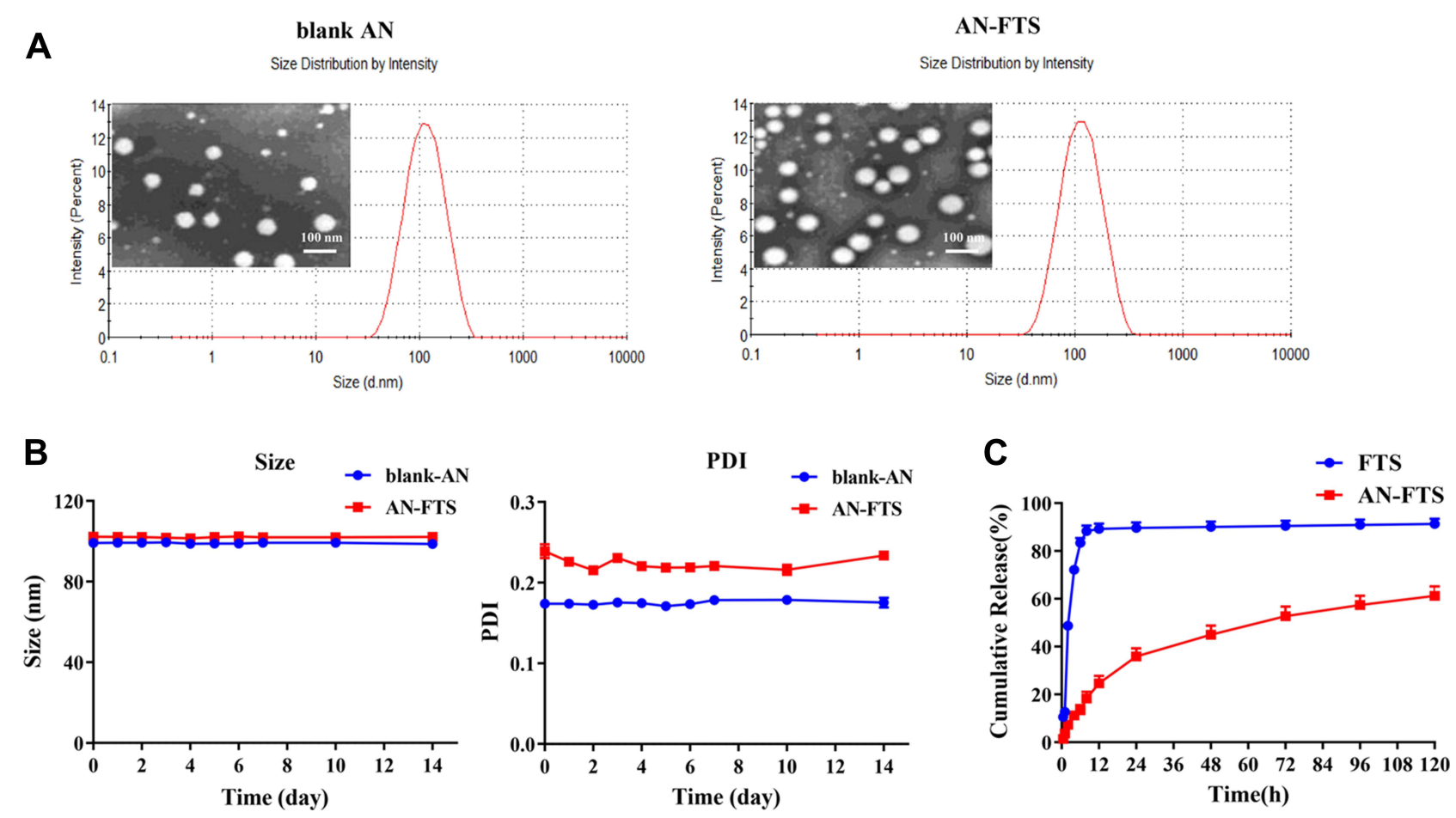

Figure I Characterization of FTS-loaded albumin nanoparticles (AN-FTS) in vitro. (A) Dynamic light scattering data and transmission electron microscope images for blank $\mathrm{AN}$ and AN-FTS. The scale bar is $100 \mathrm{~nm}$. (B) The variations in particle size and PDI of blank AN and AN-FTS at $4{ }^{\circ} \mathrm{C}$ for 14 days. All values are represented as mean \pm SEM $(n=3)$. (C) Cumulative release of free FTS and AN-FTS at $37^{\circ} \mathrm{C}$ in PBS $(\mathrm{pH} 7.4)$ containing tween-80 $(0.2 \%$, w/w). Data are shown as mean \pm SEM $(n=3)$.

AN treatment had influence on the activation of NRK-49F cells (Figure 2A and B).

Since FTS is an antagonist of Ras, we also examine whether AN-FTS could enhance the ability to inhibit Ras. TGF- $\beta 1$ upregulated the expression of Ras, confirming that the process of myofibroblast activation is accompanied with the activation of Ras. This upregulation was remarkably inhibited by AN-FTS, whereas the same concentration of free FTS only slightly inhibited the activation of Ras (Figure 2C). Thus, the enhanced anti-fibrosis efficacy of AN-FTS may attribute to the greater inhibition of Ras.

\section{Pharmacokinetics and Biodistribution of AN-DiD in Rats}

To investigate whether the albumin nanoparticle can improve the bioavailability of drug and target delivery to the kidney, we perform the pharmacokinetics and biodistribution study using fluorescent dye DiD loaded albumin nanoparticle (AN-DiD). ${ }^{25}$ Plasma concentrations of DiD overtime in rats injected with free DiD or AN-DiD were shown in Figure 3A. Levels of AN-DiD were significantly higher than those of free DiD at all time points. Compared with free DiD, AN-DiD showed greatly higher peak concentration $\left(\mathrm{C}_{\max }\right)$ and area under the curve $\left(\mathrm{AUC}_{0-\mathrm{t}}\right)$ (both $P<0.001)$, as well as slightly longer half-life $\left(\mathrm{t}_{1 / 2}\right)$ (Figure 3B).

The biodistribution studies of AN-DiD were conducted in UUO-induced renal fibrosis mice. After intravenous injection, the fluorescence intensity of AN-DiD was significantly higher than free DiD in UUO-induced obstruction kidney, while the fluorescence intensity of AN-DiD was only slightly higher, yet also statistically significant, than of free DiD in the contralateral normal kidney (Figure 3C and D). These encouraging results suggest that albumin nanoparticles may increase the therapeutic efficacy of the entrapped drug.

Table I Characteristics of Blank AN and AN-FTS $(n=3$, Mean \pm SEM)

\begin{tabular}{|l|c|c|c|c|c|}
\hline & Size $(\mathbf{n m})$ & PDI & Zeta Potential (mV) & EE (\%) & DLC (\%) \\
\hline AN & $99.12 \pm 0.87$ & $0.17 \pm 0.07$ & $17.17 \pm 0.39$ & - & - \\
AN-FTS & $100.60 \pm 1.12$ & $0.24 \pm 0.02$ & $18.99 \pm 0.41$ & $98.60 \pm 1.21$ & $2.31 \pm 0.15$ \\
\hline
\end{tabular}


A a-SMA

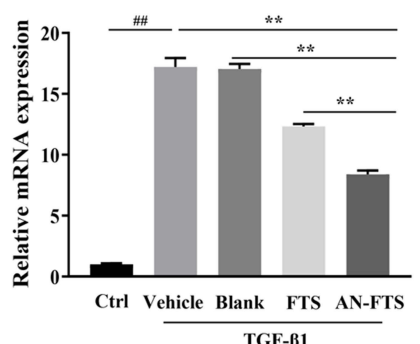

Col3a1

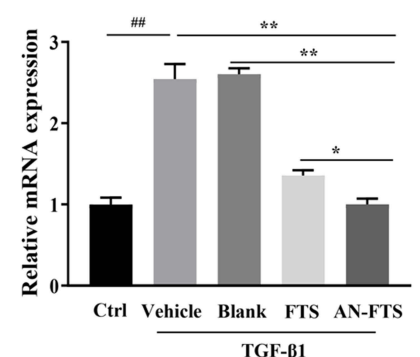

B

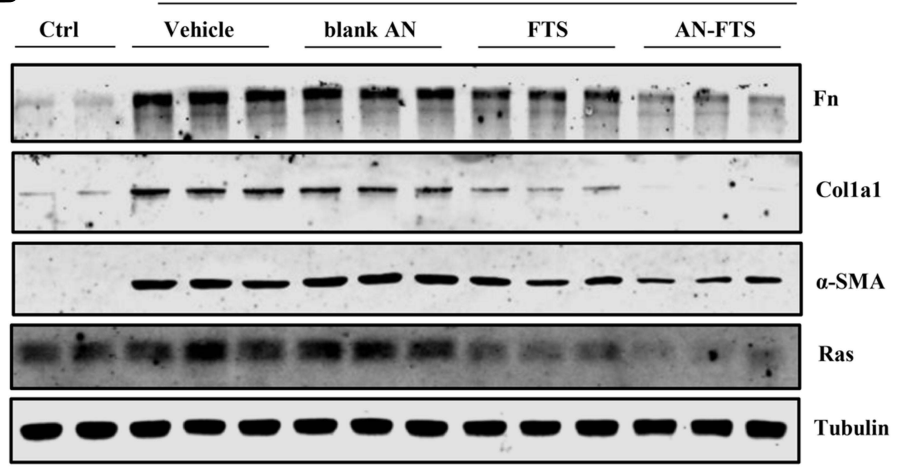

Col1a2

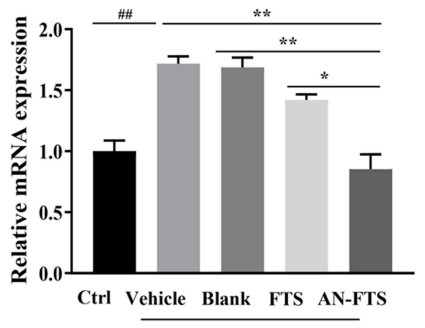

Fn

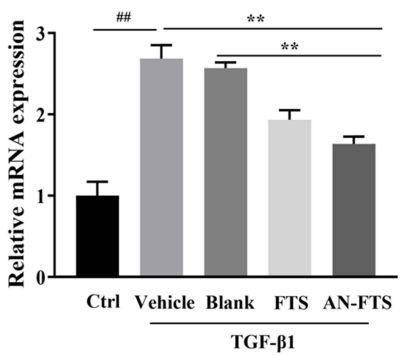

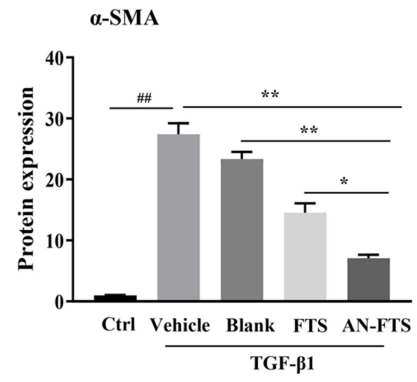

C H-Ras

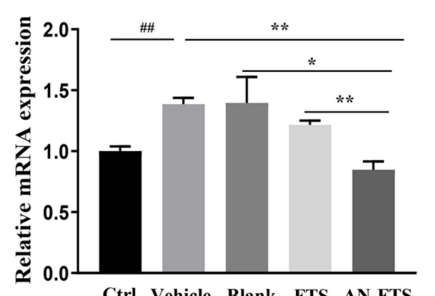

Ctrl Vehicle Blank FTS AN-FTS

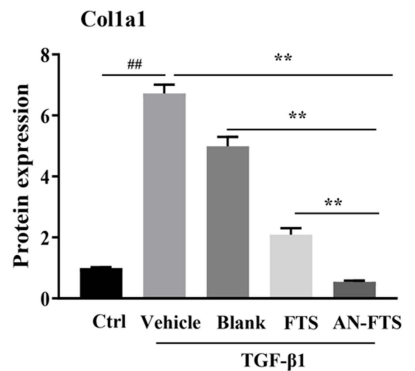

K-Ras

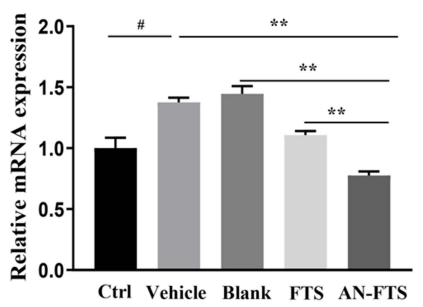

Ras

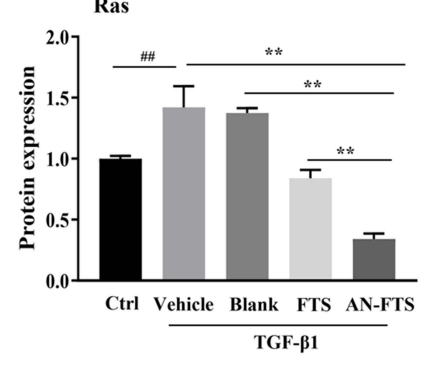

N-Ras

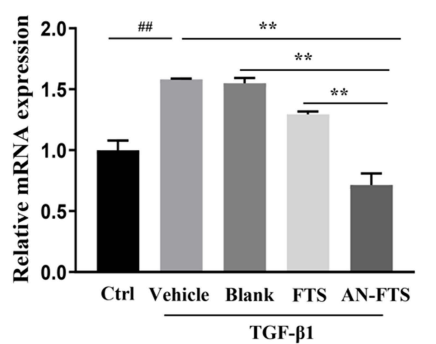

Figure 2 AN-FTS inhibited TGF- $\beta$ I-induced activation of NRK-49F cells. NRK-49F cells were treated with free FTS and AN-FTS (20 $\mu M$ of FTS) in the presence of 2 ng/mL TGF- $\beta$ I for 48 h. (A) Relative mRNA expression of $\alpha$-SMA, collagen, Pai-I and Fn in NRK-49F cells treated with free FTS and AN-FTS. (B) Protein expression of $\alpha-S M A$, Collal and Fn in NRK-49F cells treated with free FTS and AN-FTS. (C) Relative mRNA expression of H-Ras, K-Ras and N-Ras. Data are mean \pm SEM ( $n=3$ ). ${ }^{\#} P<0.01$ compared with control (ctrl) group, $* P<0.05$ and $* * P<0.01$ compared with AN-FTS group. 
A

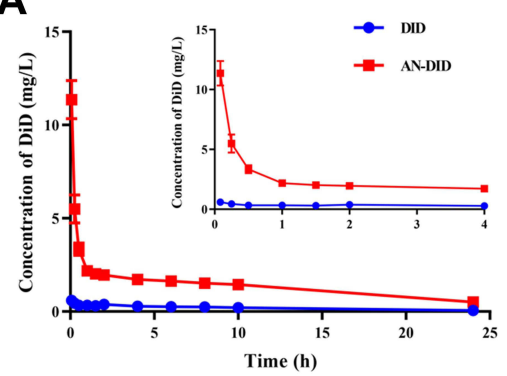

B

\begin{tabular}{ccc}
\hline PK Parametes & DID & AN-DID \\
\hline Cmax $(\mathrm{mg} / \mathrm{L})$ & $0.629 \pm 0.183$ & $12.18 \pm 1.681^{* *}$ \\
$\mathbf{t}_{1 / 2}(\mathrm{~h})$ & $9.777 \pm 3.032$ & $10.71 \pm 2.679$ \\
$\mathrm{AUC0}-\mathrm{t}(\mathrm{mg} / \mathrm{L} * \mathrm{~h})$ & $5.515 \pm 1.827$ & $34.10 \pm 3.149^{* *}$ \\
\hline
\end{tabular}

C

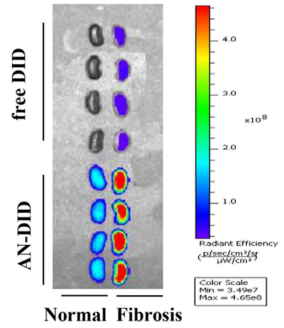

D

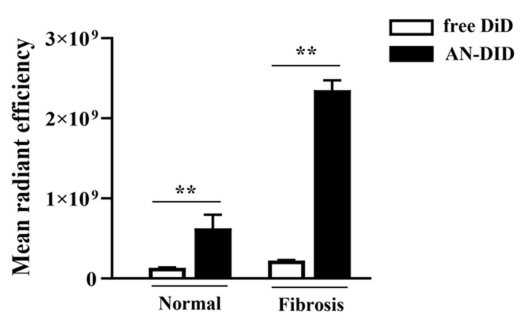

Figure 3 Pharmacokinetic profiles and in vivo distribution of AN-DiD. (A) Plasma concentration-time curve of free DiD and AN-DiD after i.v. administration in rats. (B) Pharmacokinetic parameters of free DiD and AN-DiD. AUC, area under the concentration-time curve; $t_{1 / 2}$, half-life. Values are presented as mean $\pm S E M(n=6)$, $* * P<0.01$. (C) Ex vivo images of kidneys tripped from UUO mice at $4 \mathrm{~h}$ after injection of free DiD and AN-DiD. (D) Semi-quantitative fluorescence intensity of kidneys. Data are mean $\pm \operatorname{SEM}(n=4)$, **P $<0.01$.

\section{AN-FTS Alleviates UUO-Induced Renal Injury and Inflammation}

To assess the anti-fibrosis potential of AN-FTS in vivo, the UUO mouse model was used to generate kidney fibrosis. On the 2nd day post UUO surgery, mice were intravenously injected with different formulations of FTS. UUO operation leads to tubular injury and hydronephrosis, resulting in obstructed weight gain, which AN-FTS showed a reverse effect (Figure 4A). AN-FTS improved the renal function to a much greater extent than free FTS, based on serum creatinine and urea nitrogen levels (Figure 4B and C). Hematoxylin-eosin staining indicated severe inflammatory cell infiltrations and glomerular atrophy in UUO mice. Free FTS treatment moderately improved this pathologic condition, but AN-FTS treatment showed substantial improvement (Figure 4D). AN-FTS treatment also reduced UUO-induced tubular dilatation and tubule brush border disruption more effectively than free FTS, based on periodic acid Schiff staining (Figure 4D).

Consistent with these histology results, AN-FTS obviously inhibited the mRNA expression of the inflammatory cytokines such as MCP-1, TNF- $\alpha$, F4/80 and IL1- $\beta$ (Figure $4 \mathrm{E}$ ). It has been reported that the numbers of $\mathrm{CD}^{+}$ and $\mathrm{CD} 68^{+}$cells in the kidney were significantly induced by UUO surgery, indicating the immune system in kidney was activated. ${ }^{30}$ Therefore, we also detected the CD3 and CD68 expression in the obstructed kidney. Our results showed that FTS treatments could significantly inhibit the expression of CD3 and CD68 at the mRNA level, and the inhibitory effect of AN-FTS was much stronger than that of free drugs (Figure 4E). Neither vehicle nor blank AN showed therapeutic potential for UUO-induced renal injury and inflammation (Figure 4A-E). Taken together, these results demonstrated that AN-FTS could effectively alleviate UUO-induced renal injury and inflammation.

\section{AN-FTS Alleviates UUO-Induced Renal Fibrosis}

Next, we evaluated the therapeutic efficacy of AN-FTS for UUO-induced renal fibrosis. Masson staining, which is stained positive for collagen to assess tissue fibrosis, of UUO mice kidney showed moderate improvement after free FTS treatment but substantial improvement after ANFTS treatment (Figure 5A). Consistently, AN-FTS alleviated UUO-induced elevated hydroxyproline content greater than free FTS (Figure 5B).

In UUO mice, the mRNA expression of $\alpha$-SMA, collagen, Fn, Timp-1, and Pai-1 was remarkably upregulated. After being treated with FTS, the AN-FTS group showed much stronger inhibitory effects on these fibrotic genes than the free drug group (Figure 5C and D). Consistent with the mRNA results, AN-FTS significantly inhibited 
A

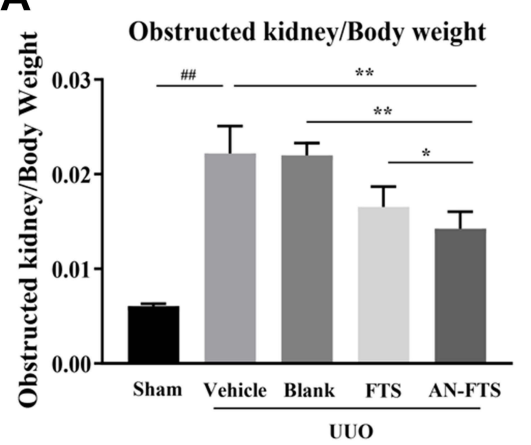

B

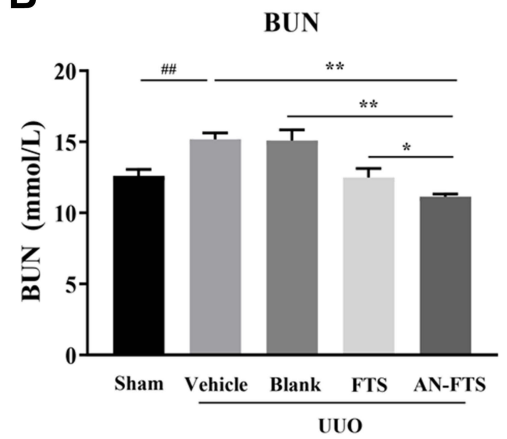

C

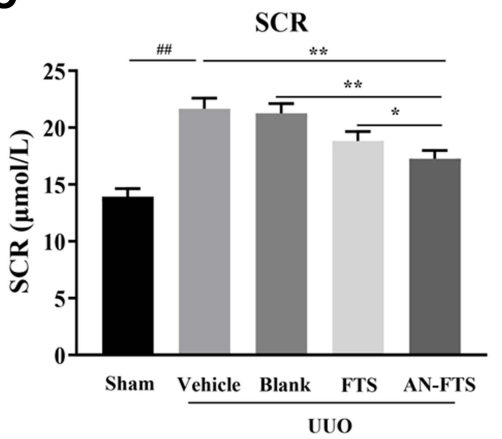

D

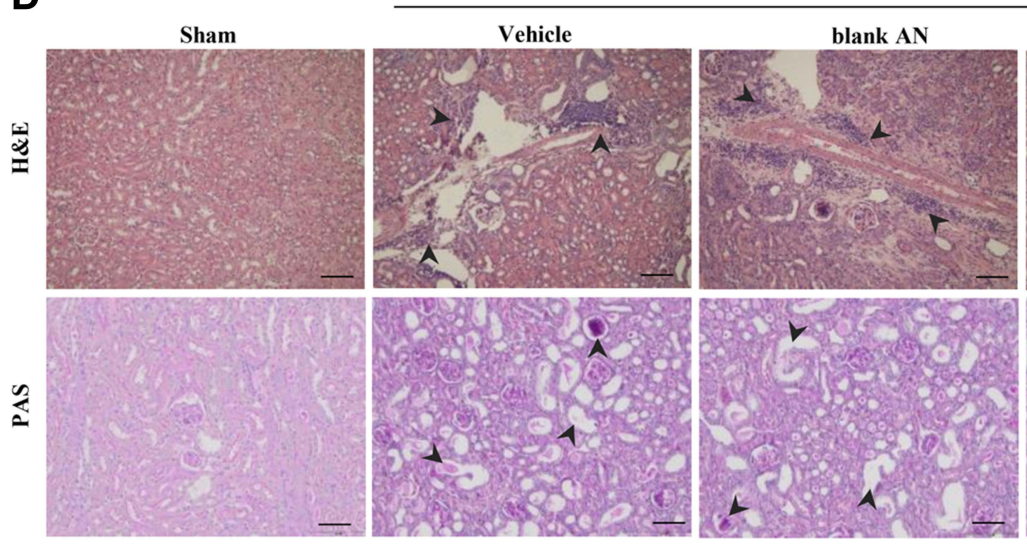

UUO

E

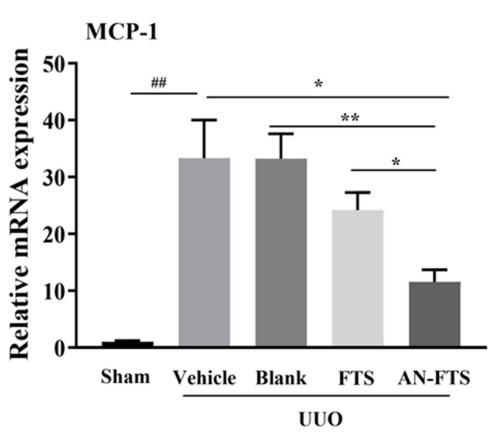

IL-1 $\beta$

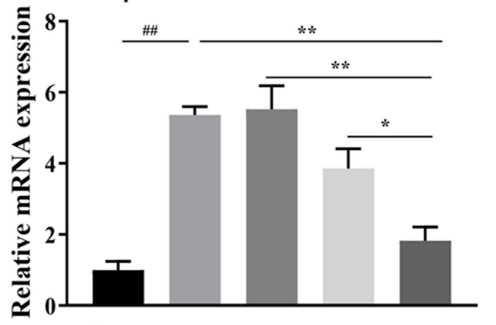

Sham Vehicle Blank FTS AN-FTS

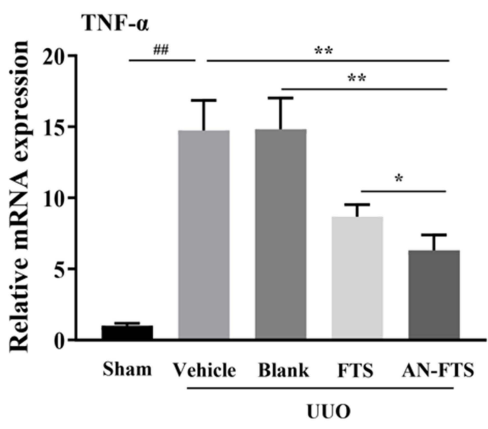

CD3

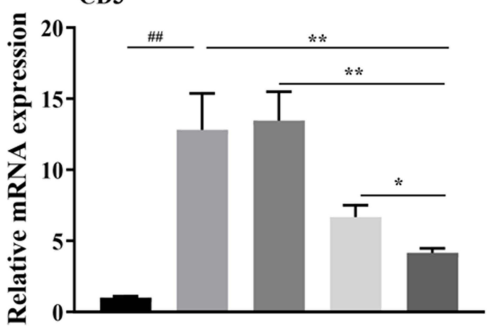

Sham Vehicle Blank FTS AN-FTS
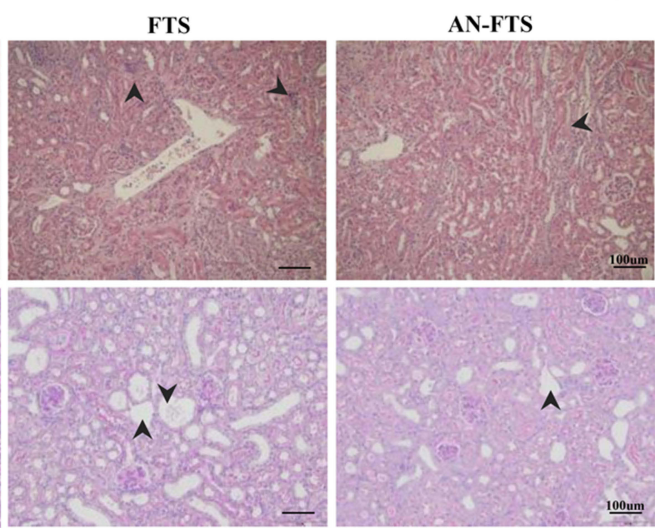

UUO

UUO
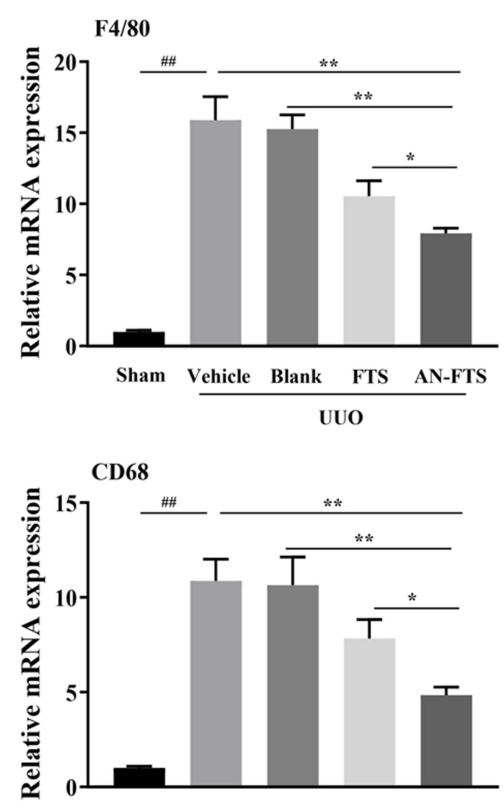

Sham Vehicle Blank FTS AN-FTS

Figure 4 AN-FTS reduced renal injury and inflammation in UUO mice. (A) Weight ratio of kidney (weight of obstructed kidney/body weight). (B) Blood urea nitrogen (BUN) levels. (C) Serum creatinine (SCR) levels. (D) H\&E and periodic acid-Schiff (PAS) staining of kidney sections. Scale bar is I00 $\mu$ m. The arrows indicated the damaged areas. (E) The relative mRNA levels of MCPI, TNF- $\alpha, F 4 / 80, I L-I \beta, C D 3$ and CD68 in kidney of sham and UUO mice. Data are mean \pm SEM ( $n=6-7)$. ${ }^{\#} P<0.01$ compared with sham group, $* P<0.05$ and $* * P<0.01$ compared with AN-FTS group. 


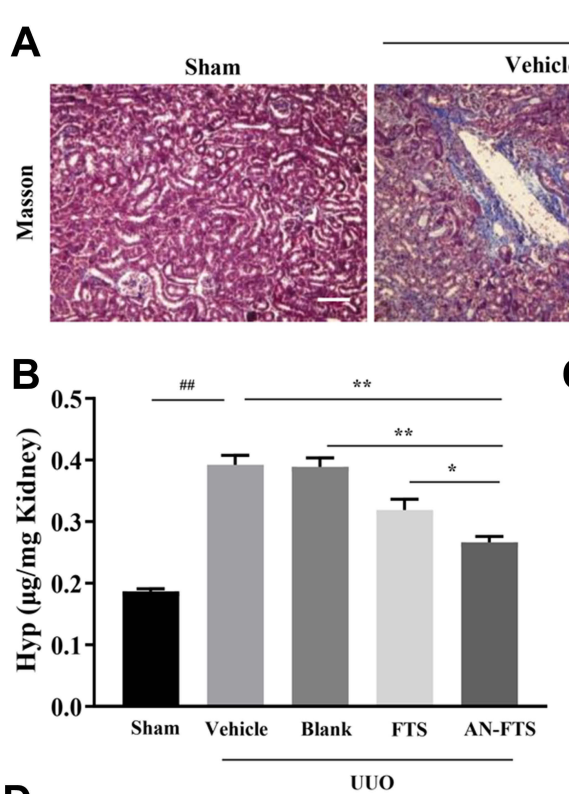

D

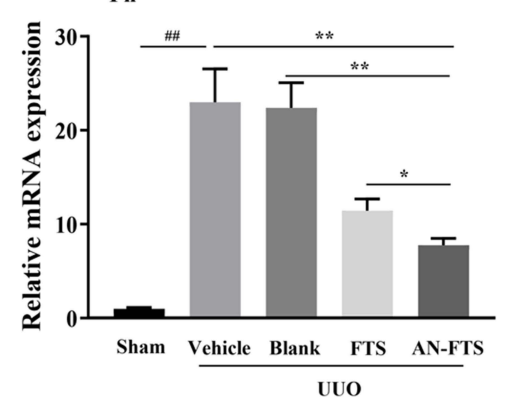

\section{C a-SMA}

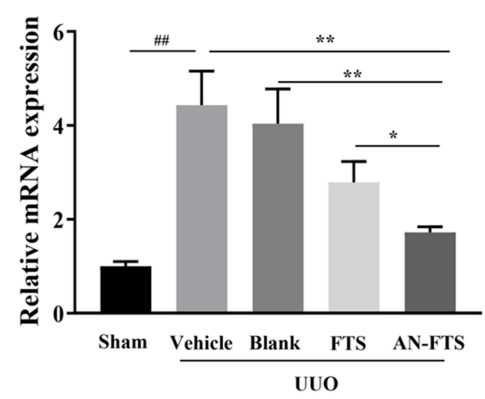

Timp-1

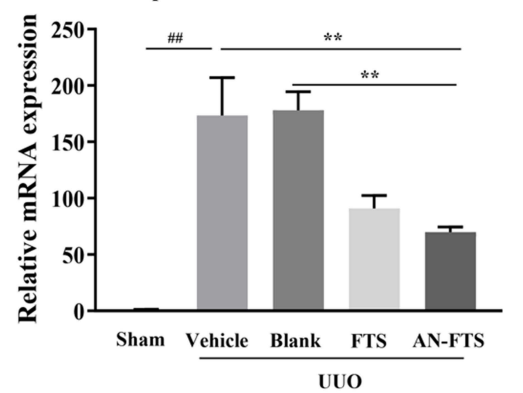

UUO
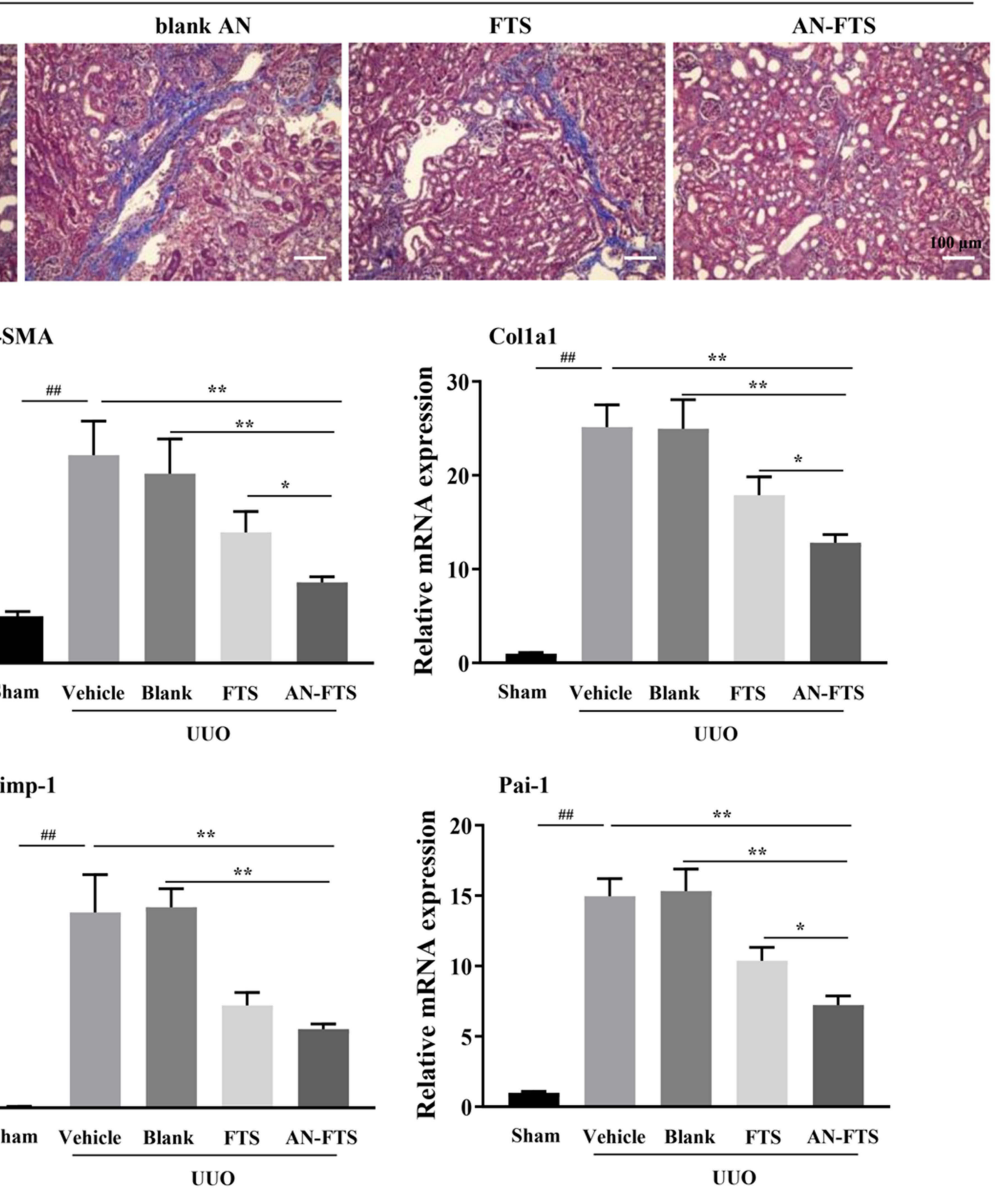

E

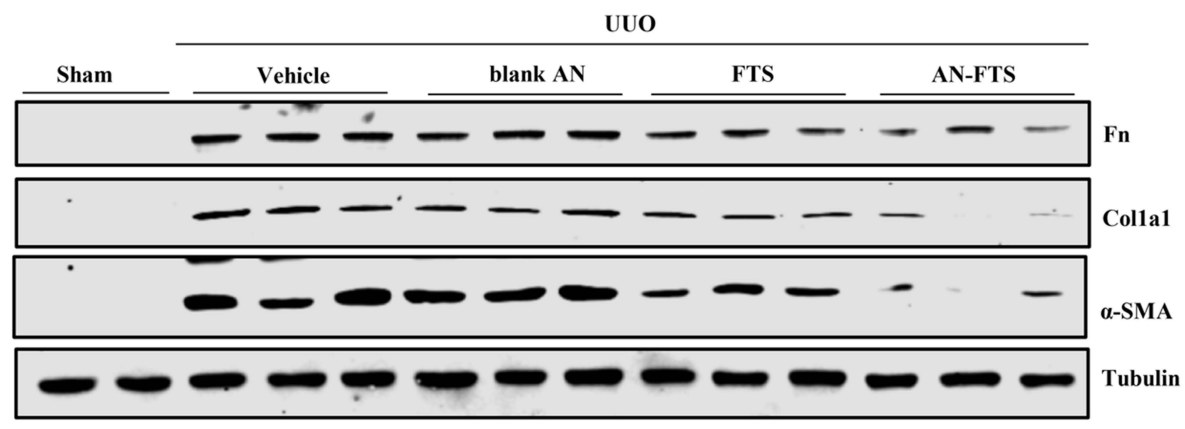

F $\quad$ Sham

UUO
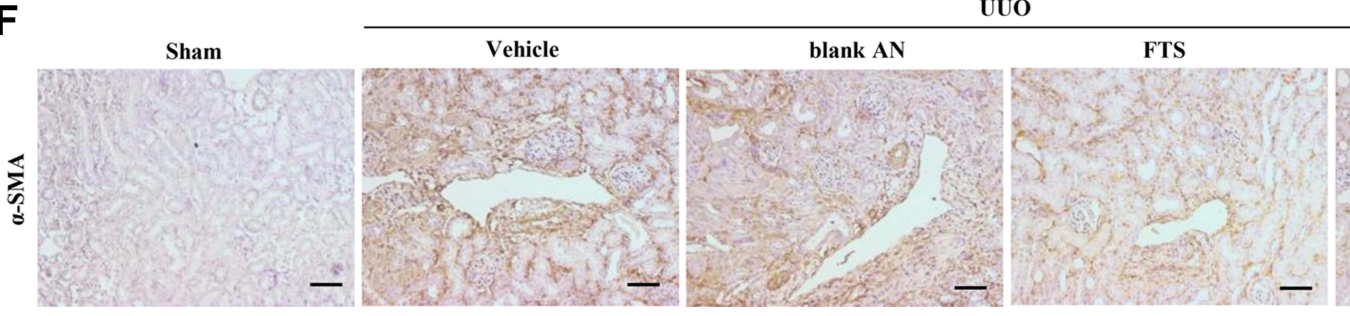

AN-FTS

Figure 5 AN-FTS attenuated renal fibrosis in UUO mice. (A) Masson staining of kidney sections. Scale bar is $100 \mu$ m. (B) Hydroxyproline content in kidney of mice. (C and D) Renal mRNA expression of $\alpha$-SMA and Collal (C), and Fn, Timp-I and Pai-I (D). (E) Protein expression of $\alpha$-SMA, Collal and Fn in kidney tissues of mice. (F) Immunohistochemistry staining of $\alpha$-SMA in kidney sections. Scale bar is $100 \mu \mathrm{m}$. Data are mean \pm SEM $(n=6-7)$. $P<0.01$ compared with Sham group, $* P<0.05$ and $* * P<$ 0.01 compared with AN-FTS group. 
the UUO-induced protein expression of $\alpha$-SMA, Colla1 and Fn (Figure 5E and Supplementary Figure 1). Additionally, $\alpha$-SMA immunohistochemistry staining further confirmed that AN-FTS inhibited UUO-induced renal fibrosis more effectively than free drug (Figure 5F). Neither vehicle nor blank AN showed therapeutic potential for UUO-induced renal fibrosis. Taken together, these results suggested that AN-FTS could effectively ameliorate renal fibrosis in UUO mice.

\section{AN-FTS Alleviates Renal Fibrosis by Inhibiting Ras/Ras I/p38 MAPK Pathway in UUO Mice}

As FTS is a synthetic small molecule antagonist of Ras gene, we first examined whether AN-FTS could inhibit the Ras gene expression in vivo. UUO upregulated the mRNA expression of K-Ras and N-Ras, and these were moderately improved by free FTS, but substantially reversed by AN-FTS (Figure 6A). Consistent with the mRNA results, immunohistochemistry staining of Ras further confirmed that AN-FTS showed more pronounced inhibitory effects on UUO-induced Ras activation than the free drug in the kidney (Figure 6B).

Epithelial mesenchymal transition (EMT) plays an important role in the occurrence and progression of renal fibrosis. ${ }^{4}$ Thus, we examined the efficacy of AN-FTS in renal EMT process. UUO upregulated the mRNA expression of vimentin and snail, and downregulated the mRNA expression of E-cadherin, suggesting UUO successfully induced renal EMT. Compared to free FTS, AN-FTS treatment significantly reduced the expression of vimentin and snail, and elevated the expression of E-cadherin (Figure 7A). Consistent with these mRNA results, ANFTS remarkably decreased the UUO-induced vimentin and snail protein expression, and increased the E-cadherin protein expression (Figure 7B and Supplementary Figure 2). These results suggested that AN-FTS can inhibit UUO-induced EMT process in mouse kidney.

It is reported that EMT process in the fibrotic kidney can be inhibited via the Ras/Raf1/p38 MAPK signaling pathway. $^{7,12}$ Therefore, we explored whether AN-FTS could inhibit renal EMT process via Ras/Raf1/p38 MAPK signaling pathway. Results of Western blot showed that UUO significantly induced phosphorylation of Raf1 and p38. AN-FTS inhibited UUO-induced Raf1 and p38 phosphorylation more effectively than the free drug (Figure 7C and D). The immunofluorescence staining of p-Raf1 and p-p38 also confirmed this result (Figure 7E). Taken together, these results demonstrated the anti-fibrosis effects of AN-FTS was associated with inhibition in renal EMT process via Ras/Raf1/p38 signaling pathway.

\section{Discussion}

In the present study, we loaded Ras antagonist FTS into bovine serum albumin nanoparticle (AN-FTS) with size of $100 \mathrm{~nm}$ to improve its poor water solubility and low bioavailability. In vitro, AN-FTS showed stronger inhibitory efficacy on NRK-49F cells activation than free FTS. In vivo, AN-FTS significantly improved the bioavailability of FTS and preferentially accumulated in fibrotic kidney. After systemic administration, AN-FTS alleviated renal
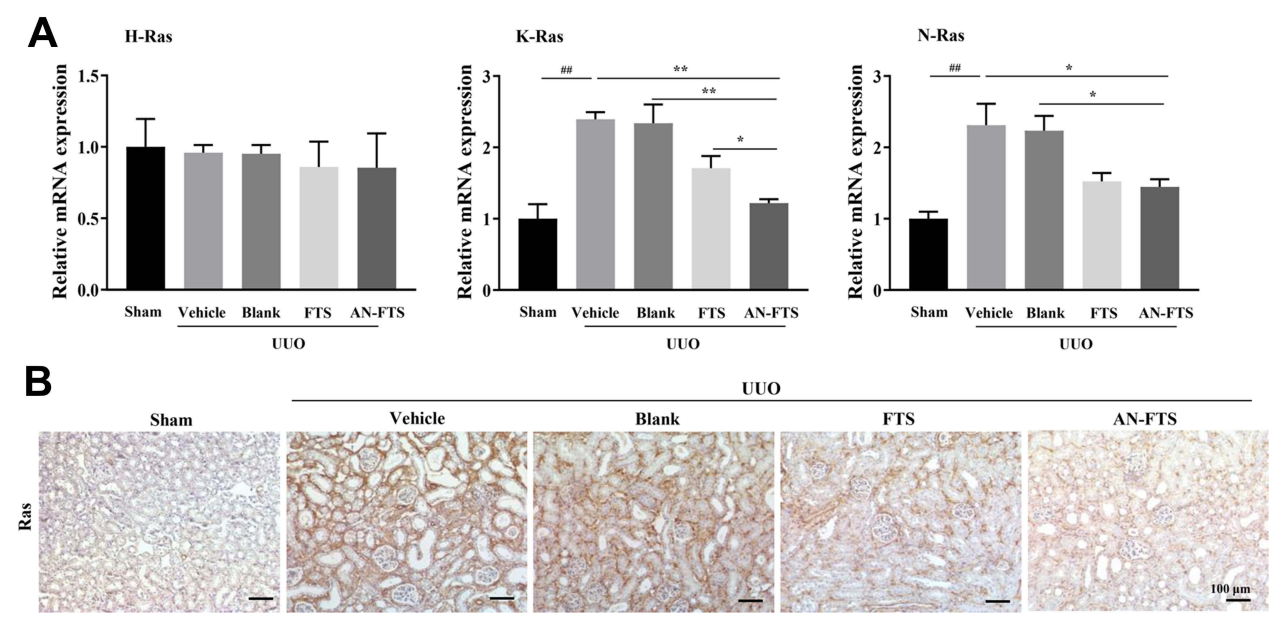

Figure 6 AN-FTS inhibited UUO-induced Ras expression in kidney of mice. (A) The relative expression of H-Ras, K-Ras and N-Ras in kidney tissues of mice. (B) Immunohistochemistry staining of Ras in kidney sections. Data are mean \pm SEM $(n=6-7)$. $P<0.0$ I compared with Sham group, $* P<0.05$ and $* * P<0.0$ I compared with AN-FTS group. 


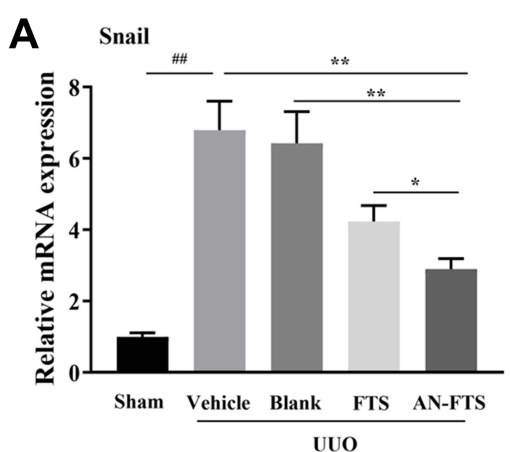

B
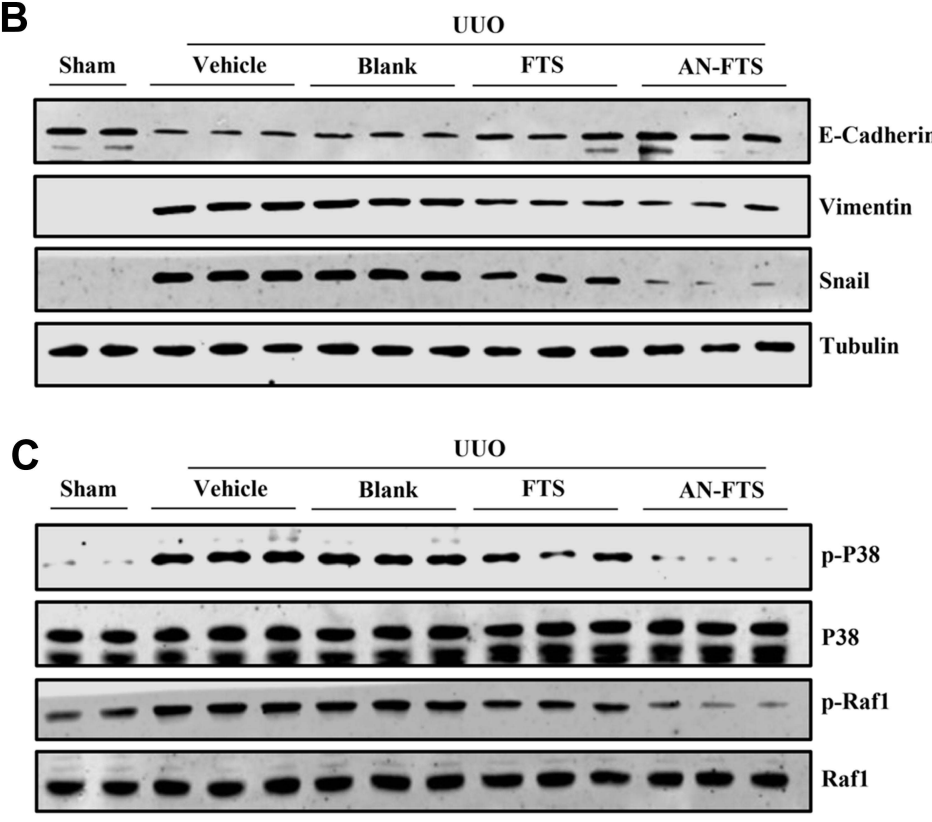

Vimentin

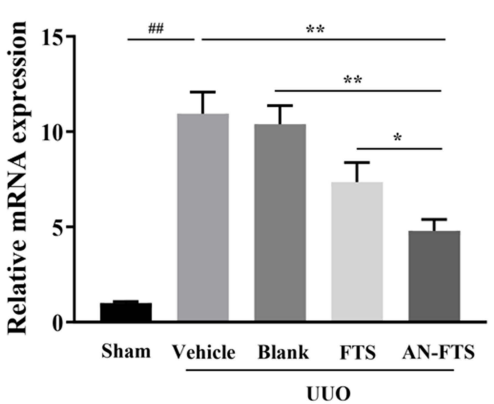

E-cadherin

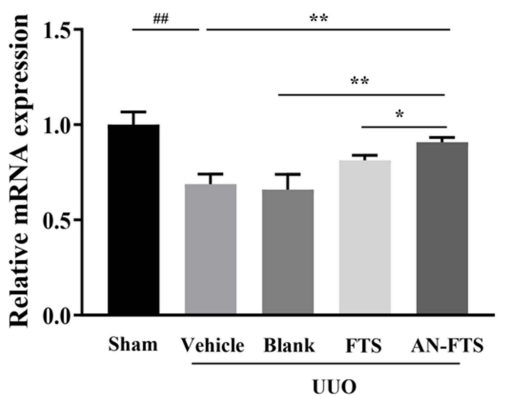

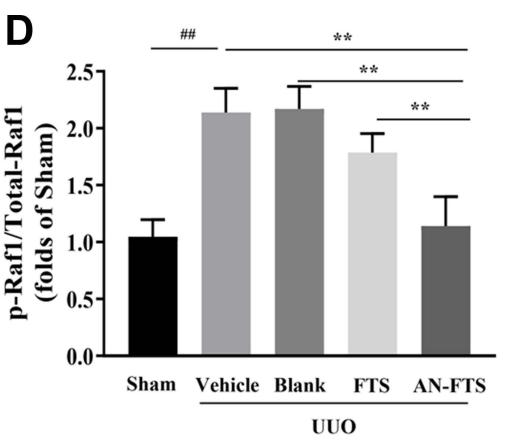

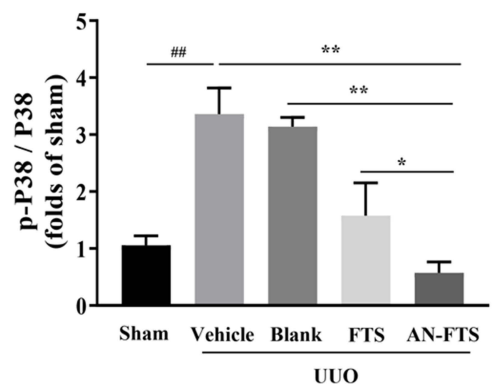

E

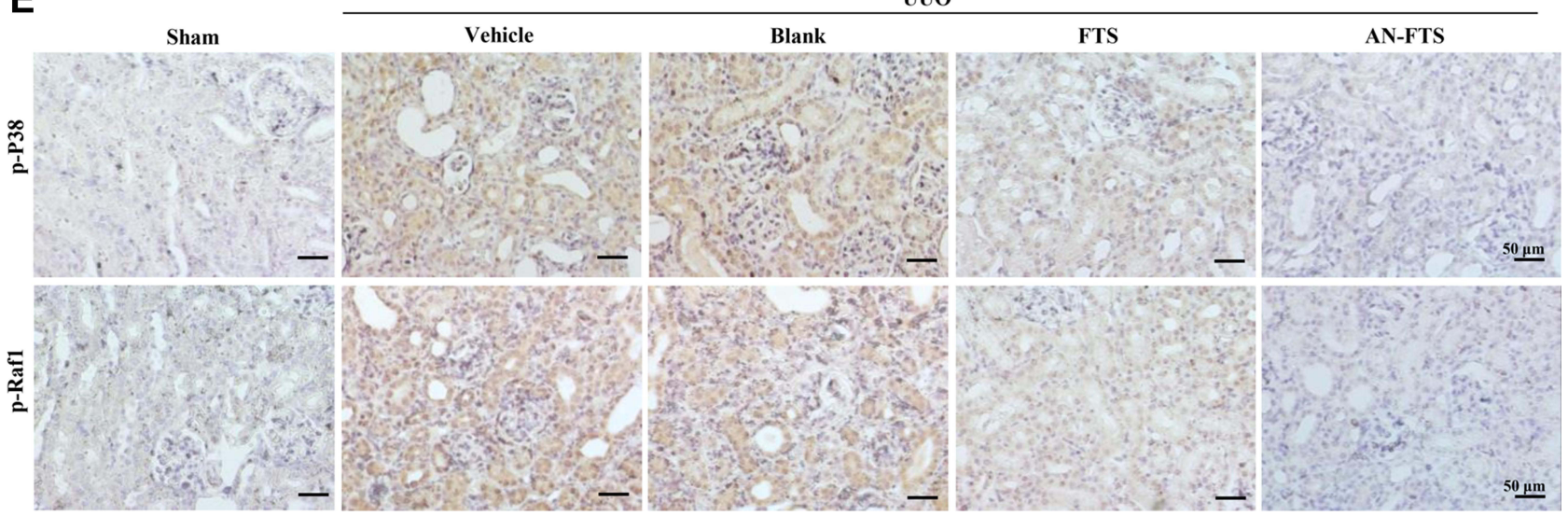

Figure 7 AN-FTS suppressed EMT process by inhibiting Raf/P38 signaling pathway in kidney of UUO mice. (A) Renal mRNA expression of snail, vimentin and E-cadherin in indicated groups. Data are mean \pm SEM $(n=6-7)$. (B) Protein expression of E-cadherin, vimentin and snail in kidney tissues of sham and UUO mice. (C) Protein expression of phospho-Rafl and phospho-P38 in kidney tissues of sham and UUO mice. (D) Quantification of Western blot analysis in (C). (E) Immunohistochemistry staining of p-Rafl and $\mathrm{P}-\mathrm{P} 38$ in kidney sections. Data are mean \pm SEM $(n=3) .{ }^{\#} \mathrm{P}<0.01$ compared with sham group, $* \mathrm{P}<0.05$ and $* * \mathrm{P}<0.01$ compared with AN-FTS group.

injury, inflammation and fibrosis more effectively than the free drug. Mechanistically, the anti-fibrosis effects of ANFTS were associated with greater inhibition effects in renal EMT process via the Ras/Raf1/p38 signaling pathway.
FTS, a specific inhibitor of proto-oncogene Ras, has shown therapeutic potential for renal fibrosis. However, FTS is insoluble in water and has low selectivity in the body. ${ }^{23,24}$ It is widely distributed in the body and easy to 
be discharged through the glomerular filtration and excreted in urine, which limit its clinical application. In the past decades, it is well documented that nanoparticles have been used as a promising drug delivery system for a wide range of therapeutic agents. ${ }^{31}$ Here, we selected $\mathrm{AN}$ as the nanocarrier system for FTS mainly due to following reasons. AN were made from bovine serum albumin, with the characters being biodegradable, nontoxic and non-immunogenic, which can facilitate rapid translation into the clinic. As a hydrophobic drug, FTS can be easily encapsulated into AN due to the highbinding affinity to albumin. ${ }^{18-21}$ Besides, the preparation process of albumin nanoparticles is simple and easy to scale up production. Therefore, we encapsulated FTS into bovine serum albumin nanoparticles to increase the water-solubility and improve the bioavailability.

Nanoparticle size is an important determinant of circulation time and tissue deposition after administration. The mesangial space is clearly accessible through the glomerular vascular fenestrations, which specifically have a relatively wide width of 70 to $130 \mathrm{~nm}^{22}$ Based on the special physiological structure of the kidney, several preclinical research studies described nanocarriers appeared to selectively target kidneys. Gold nanoparticles with a defined size of $\sim 75 \pm 25 \mathrm{~nm}$ were shown to specifically accumulate in mesangial cells in mice. ${ }^{32-34}$ Recently, Guo et al showed that albumin nanoparticles with a particle size of 70-130 nm can be passively targeted to the kidney. Particularly, AN with a particle size of $95 \mathrm{~nm}$ achieved the highest accumulation in the kidney. ${ }^{22}$ In the present study, through optimization of preparation, we successfully prepared AN-FTS with a size of $100 \mathrm{~nm}$. Biodistribution study confirmed that our AN-FTS could specifically accumulate in UUO-induced fibrotic kidney.

Our results showed that AN-FTS presented greater anti-fibrosis activity than free FTS both in vitro and in vivo. AN-FTS was superior to free drug in inhibiting renal fibroblast cells NRK-49F activation, as well as protecting UUO-induced renal fibrosis mouse model against renal injury, inflammation, and fibrosis. The enhanced therapeutic efficacy of AN-FTS may involve multiple mechanisms. After being entrapped in AN, FTS showed slowly release profiles as shown in in vitro release assays, resulting in longer retention time in the body. The ability of albumin nanoparticles to solubilize FTS translated to greater bioavailability, as evidenced by pharmacokinetic studies, which showed significantly higher $\mathrm{C}_{\max }$ and $\mathrm{AUC}_{0-\mathrm{t}}$ of AN-FTS. Furthermore, the passive target ability of AN-FTS in fibrotic kidney significantly increased the drug concentration in diseased kidney tissues, thus enhancing the therapeutic effects.

The pathogenesis of renal fibrosis is very complex, among which EMT plays an important role in its occurrence and progression. ${ }^{4}$ Previous studies have reported that the proto-oncogene Ras is crucial for the UUO-induced EMT in renal fibrosis. ${ }^{35}$ Moreover, the EMT progression in renal fibrosis can be suppressed by inhibiting the activation of Ras/Raf1/p38 MAPK signaling pathway. ${ }^{7}$ Our results showed that AN-FTS could inhibit the EMT process in kidney. Inhibition of Ras/Raf1/p38 pathway by AN-FTS administration seemed to be the underlying mechanism in suppressing the renal EMT, and thus leading to alleviate renal fibrosis. More in-depth molecular mechanisms explorations will be focused on this part in our future study.

In conclusion, encapsulating the water-insoluble drug FTS into albumin nanoparticles remarkably improved its solubility and pharmacokinetic properties. FTS-loaded albumin nanoparticles exhibited a stronger anti-fibrosis effect than free FTS both in vitro and in vivo, by inhibiting renal EMT process via Ras/Raf1/P38 MAPK signaling pathway. These results manifested that the AN-FTS nanoparticle system is effective in delivering FTS to fibrotic kidney and showed superior therapeutic efficacy for renal fibrosis.

\section{Acknowledgments}

This work was supported by the National Natural Science Foundation of China (81870599, 81873662 and 81930020), research funding from Sichuan Province (2020YFS0064).

\section{Disclosure}

The authors declare no competing interests.

\section{References}

1. Ammirati AL. Chronic kidney disease. Rev Assoc Med Bras. 2020;66 (Suppl 1):s03-s9. doi:10.1590/1806-9282.66.S1.3

2. Hallan SI, Ovrehus MA, Romundstad S, et al. Long-term trends in the prevalence of chronic kidney disease and the influence of cardiovascular risk factors in Norway. Kidney Int. 2016;90(3):665-673. doi:10.1016/j.kint.2016.04.012

3. Humphreys BD. Mechanisms of renal fibrosis. Annu Rev Physiol. 2018;80(1):309-326. doi:10.1146/annurev-physiol-022516-034227

4. Lovisa S, Zeisberg M, Kalluri R. Partial epithelial-to-mesenchymal transition and other new mechanisms of kidney fibrosis. Trends Endocrinol Metab. 2016;27(10):681-695. doi:10.1016/j.tem.2016.06.004 
5. Tan X, He W, Liu Y. Combination therapy with paricalcitol and trandolapril reduces renal fibrosis in obstructive nephropathy. Kidney Int. 2009;76(12):1248-1257. doi:10.1038/ki.2009.346

6. Xu J, Lamouille S, Derynck R. TGF-beta-induced epithelial to mesenchymal transition. Cell Res. 2009;19(2):156-172. doi:10.1038/cr.2009.5

7. Su J, Morgani SM, David CJ, et al. TGF-beta orchestrates fibrogenic and developmental EMTs via the RAS effector RREB1. Nature. 2020;577(7791):566-571. doi:10.1038/s41586-019-1897-5

8. Samatar AA, Poulikakos PI. Targeting RAS-ERK signalling in cancer: promises and challenges. Nat Rev Drug Discov. 2014;13 (12):928-942. doi:10.1038/nrd4281

9. Rodríguez-Peña AB, Santos E, Arévalo M, López-Novoa JM. Activation of small GTPase Ras and renal fibrosis. J Nephrol. 2005;18:341-349.

10. Grande MT, Fuentes-Calvo I, Arevalo M, et al. Deletion of H-Ras decreases renal fibrosis and myofibroblast activation following ureteral obstruction in mice. Kidney Int. 2010;77(6):509-518. doi:10.1038/ ki. 2009.498

11. Newbury LJ, Wang JH, Hung G, Hendry BM, Sharpe CC. Inhibition of Kirsten-Ras reduces fibrosis and protects against renal dysfunction in a mouse model of chronic folic acid nephropathy. Sci Rep. 2019;9 (1):14010. doi:10.1038/s41598-019-50422-7

12. Gaestel M. MAPK-activated protein kinases (MKs): novel insights and challenges. Front Cell Dev Biol. 2015;3:88. doi:10.3389/ fcell.2015.00088

13. Marom M, Haklai R, Ben-Baruch G, Marciano D, Egozi Y, Kloog Y. Selective inhibition of Ras- dependent cell growth by farnesylthiosalisylic acid. J Biol Chem. 1995;270(38):22263-22270. doi:10.1074/ jbc. 270.38 .22263

14. Menachem A, Bodner O, Pastor J, Raz A, Kloog Y. Inhibition of malignant thyroid carcinoma cell proliferation by Ras and galectin-3 inhibitors. Cell Death Discov. 2015;1(1):15047. doi:10.1038/ cddiscovery. 2015.47

15. Reif S, Weis B, Aeed H, et al. The Ras antagonist, farnesylthiosalicylic acid (FTS), inhibits experimentally-induced liver cirrhosis in rats. J Hepatol. 1999;31(6):1053-1061. doi:10.1016/s0168-8278(99)80318-3

16. Reif S, Aeed H, Shilo Y, et al. Treatment of thioacetamide-induced liver cirrhosis by the Ras antagonist, farnesylthiosalicylic acid. J Hepatol. 2004;41(2):235-241. doi:10.1016/j.jhep.2004.04.010

17. Clarke HC, Kocher HM, Khwaja A, Kloog Y, Cook HT, Hendry BM. Ras antagonist farnesylthiosalicylic acid (FTS) reduces glomerular cellular proliferation and macrophage number in rat thy-1 nephritis. $J \quad$ Am Soc Nephrol. 2003;14(4):848-854. doi:10.1097/01. asn.0000057543.55318.8b

18. Irvine DJ, Dane EL. Enhancing cancer immunotherapy with nanomedicine. Nat Rev Immunol. 2020;20(5):321-334. doi:10.1038/ s41577-019-0269-6

19. Elzoghby AO, Samy WM, Elgindy NA. Albumin-based nanoparticles as potential controlled release drug delivery systems. J Control Release. 2012;157(2):168-182. doi:10.1016/j.jconrel.2011.07.031

20. Montero AJ, Adams B, Diaz-Montero CM, Gluck S. Nab-paclitaxel in the treatment of metastatic breast cancer: a comprehensive review. Expert Rev Clin Pharmacol. 2011;4(3):329-334. doi:10.1586/ ecp. 11.7

International Journal of Nanomedicine

\section{Publish your work in this journal}

The International Journal of Nanomedicine is an international, peerreviewed journal focusing on the application of nanotechnology in diagnostics, therapeutics, and drug delivery systems throughout the biomedical field. This journal is indexed on PubMed Central, MedLine, CAS, SciSearch ${ }^{\mathbb{B}}$, Current Contents ${ }^{\mathbb{B}} /$ Clinical Medicine,
21. Kundranda MN, Niu J. Albumin-bound paclitaxel in solid tumors: clinical development and future directions. Drug Des Devel Ther. 2015;9:3767-3777. doi:10.2147/DDDT.S88023

22. Guo L, Luo S, Du Z, et al. Targeted delivery of celastrol to mesangial cells is effective against mesangioproliferative glomerulonephritis. Nat Commun. 2017;8(1):878. doi:10.1038/s41467-017-00834-8

23. Zhang X, Liu K, Huang Y, et al. Reduction-sensitive dual functional nanomicelles for improved delivery of paclitaxel. Bioconjug Chem. 2014;25(9):1689-1696. doi:10.1021/bc500292j

24. Zhang X, Lu J, Huang Y, et al. PEG-farnesylthiosalicylate conjugate as a nanomicellar carrier for delivery of paclitaxel. Bioconjug Chem. 2013;24(3):464-472. doi:10.1021/bc300608h

25. Li R, Li Y, Zhang J, et al. Targeted delivery of celastrol to renal interstitial myofibroblasts using fibronectin-binding liposomes attenuates renal fibrosis and reduces systemic toxicity. $J$ Control Release. 2020;320:32-44. doi:10.1016/j.jconrel.2020.01.017

26. Li X, Pan J, Li H, et al. DsbA-L mediated renal tubulointerstitial fibrosis in UUO mice. Nat Commun. 2020;11(1):4467. doi:10.1038/ s41467-020-18304-z

27. Chevalier RL, Forbes MS, Thornhill BA. Ureteral obstruction as a model of renal interstitial fibrosis and obstructive nephropathy. Kidney Int. 2009;75(11):1145-1152. doi:10.1038/ki.2009.86

28. Sato Y, Yanagita M. Resident fibroblasts in the kidney: a major driver of fibrosis and inflammation. Inflamm Regen. 2017;37(1):17. doi:10.1186/s41232-017-0048-3

29. Meng XM, Nikolic-Paterson DJ, Lan HY. TGF-beta: the master regulator of fibrosis. Nat Rev Nephrol. 2016;12(6):325-338. doi:10.1038/nrneph.2016.48

30. Chen DQ, Cao G, Chen H, et al. Identification of serum metabolites associating with chronic kidney disease progression and anti-fibrotic effect of 5-methoxytryptophan. Nat Commun. 2019;10(1):1476. doi:10.1038/s41467-019-09329-0

31. Reis CP, Neufeld RJ, Ribeiro AJ, Veiga F. Nanoencapsulation I. Methods for preparation of drug loaded polymeric nanoparticles. Nanomedicine. 2006;2(1):8-21. doi:10.1016/j.nano.2005.12.003

32. Choi CH, Zuckerman JE, Webster P, Davis ME. Targeting kidney mesangium by nanoparticles of defined size. Proc Natl Acad Sci U S A. 2011;108(16):6656-6661. doi:10.1073/pnas.1103573108

33. Schipper ML, Iyer G, Koh AL, et al. Particle size, surface coating, and PEGylation influence the biodistribution of quantum dots in living mice. Small. 2009;5(1):126-134. doi:10.1002/smll.200800003

34. Lahdenkari AT, Lounatmaa K, Patrakka J, et al. Podocytes are firmly attached to glomerular basement membrane in kidneys with heavy proteinuria. J Am Soc Nephrol. 2004;15(10):2611-2618. doi:10.1097/ 01.ASN.0000139478.03463.D9

35. Martinez-Salgado C, Fuentes-Calvo I, Garcia-Cenador B, Santos E, Lopez-Novoa JM. Involvement of $\mathrm{H}-$ and $\mathrm{N}$-Ras isoforms in transforming growth factor-beta1-induced proliferation and in collagen and fibronectin synthesis. Exp Cell Res. 2006;312(11):2093-2106. doi:10.1016/j.yexcr.2006.03.008
Journal Citation Reports/Science Edition, EMBase, Scopus and the Elsevier Bibliographic databases. The manuscript management system is completely online and includes a very quick and fair peer-review system, which is all easy to use. Visit http://www.dovepress.com/ testimonials.php to read real quotes from published authors.

\section{Dovepress}

